\title{
Which is the optimal management for locally advanced gastric cancer patients with TRG 0 and 1 after R0 resection?
}

\author{
Fei Ma ${ }^{1}$, Yonglei Zhang ${ }^{1}$, Liangqun Peng ${ }^{1}$, Zhandong Zhang ${ }^{1}$, Wei Yang ${ }^{1}$, Junhui Chai ${ }^{1}$, Bin Zhang ${ }^{1}$, \\ Sheqing Ji ${ }^{1}$, Yawei Hua ${ }^{1}$, Xiaobing Chen ${ }^{2}$, Suxia Luo ${ }^{2}$ \\ ${ }^{1}$ Department of General Surgery, ${ }^{2}$ Department of Digestion, The Affiliated Tumor Hospital of Zhengzhou University, Zhengzhou, China \\ Contributions: (I) Conception and design: F Ma; (II) Administrative support: S Luo; (III) Provision of study materials or patients: Y Zhang, Z Zhang, \\ B Zhang, S Ji; (IV) Collection and assembly of data: F Ma, L Peng, W Yang; (V) Data analysis and interpretation: F Ma, L Peng, Y Hua; (VI) \\ Manuscript writing: All authors; (VII) Final approval of manuscript: All authors. \\ Correspondence to: Suxia Luo. Department of Digestion, The Affiliated Tumor Hospital of Zhengzhou University, No. 127 Dongming Road, \\ Zhengzhou 450008, China. Email: zlyyluosuxia0361@zzu.edu.cn.
}

Background: Neoadjuvant chemotherapy (NAC) followed by surgery currently offers promise as a strategy for patients with locally advanced gastric cancer (GC). However, there is limited evidence to guide treatment for TRG 0 and 1 patients with locally advanced GC after R0 resection. This study set out to explore the optimal management for TRG 0 and 1 patients with locally advanced GC after R0 resection.

Methods: The retrospective data of 154 TRG 0 and 1 patients with locally advanced GC following R0 resection who were treated between January 2012 and December 2018 were collected and analyzed. The Kaplan-Meier method was used to estimate the survival rate. Multivariate analysis was performed using the Cox proportional hazards model.

Results: The median follow-up was 34.1 (range, 6.6-90.9) months. Six patients (3.9\%) were lost during follow-up. Of the 27 patients who experienced relapse, 12 died, including 2 patients who died of nonneoplastic causes. The 5-year recurrence-free survival (RFS) and 5-year overall survival (OS) were 71.6\% (95\% CI: 68.5-79.6) and 82.9\% (95\% CI: 76.9-86.1) for the whole cohort, respectively. Univariate analysis revealed that patients with carcinoembryonic antigen (CEA) $<5.0 \mathrm{ng} / \mathrm{ml}$ after NAC $(77.7 \%$ vs. $20.1 \%$, $\mathrm{P}<0.001)$, distal gastrectomy (91.7\% vs. $67.5 \%, \mathrm{P}=0.046)$ had higher 5-year RFS. Meanwhile, combined resection $(55.6 \%$ vs. $73.1 \%, \mathrm{P}=0.042$ ), major complications ( $42.7 \%$ vs. $80.50 \%, \mathrm{P}<0.001$ ), and lymph node metastasis $(\mathrm{ypN}+)(52.0 \%$ vs. $83.7 \%, \mathrm{P}<0.001)$ had lower 5 -year RFS. The multivariate analysis showed that CEA level after NAC (HR =2.876, 95\% CI: 1.051-7.872, P=0.040), major complications (HR =2.432, 95\% CI: 1.062-5.567, $\mathrm{P}=0.035)$, and lymph node metastasis (ypN+) $(\mathrm{HR}=3.183,95 \% \mathrm{CI}: 1.242-8.161, \mathrm{P}=0.016)$ were independent prognostic factors.

Conclusions: TRG 0 and 1 patients with local GC after R0 resection following NAC had a good prognosis, especially patients with CEA $<5.0 \mathrm{ng} / \mathrm{mL}$ after NAC, and those without major complications or lymph node metastasis. Monotherapy or no chemotherapy may offer options for treating TRG 0 and 1 patients without adverse prognostic factors.

Keywords: Gastric cancer (GC); neoadjuvant chemotherapy (NAC); tumor regression grading; prognosis

Submitted Feb 15, 2020. Accepted for publication Jul 22, 2020.

doi: 10.21037/atm-20-3986

View this article at: http://dx.doi.org/10.21037/atm-20-3986 


\section{Introduction}

Gastric cancer (GC) is one of the most common cancers in the world. In China, GC ranks as the second most common cancer, and occupies the same position in terms of cancerrelated mortality (1). Owing to a lack of screening, locally advanced GC comprises more than $70 \%$ of GC diagnoses in China. Radical resection offers a promising treatment strategy for patients with resectable GC. However, the outcomes for locally advanced GC patients who receive surgery are not satisfactory because of distant lymph node or distant metastases, or recurrence (2). The discovery of new treatments is essential to improving the prognosis of GC.

Since it was reported in 1989 , neoadjuvant chemotherapy (NAC) has attracted a high level of interest (3). At present, NAC followed by surgery is a helpful therapy for locally advanced GC. Many clinical investigations have shown that NAC can downstage the primary tumor, improve the $\mathrm{R} 0$ resection rate, treat potential micrometastases in advanced GC, and achieve a survival benefit (4-6). Nevertheless, many problems remain be solved in relation to NAC, including appropriate population choice, accurate preoperative staging, the choice of preoperative chemotherapy regimen, the efficacy criteria for preoperative chemotherapy, and the selection of a postoperative adjuvant chemotherapy regimen. After surgery, most patients who receive NAC receive post-operative chemotherapy, and to avoid ineffective and effective NAC, the regimen remains the same as it was before surgery.

Histologic response to NAC is useful for assessing the sensitivity of the tumor to chemotherapy and predicting prognosis in patients. Several studies have proposed four tumor regression grade (TRG) systems, the criteria of which are shown in Table 1. In 1994, a five-layered TRG system for esophageal cancer was first proposed by Mandard et al. and has since been widely applied for malignancies of the digestive system (7). Because patients with TRG 1 and 2 demonstrate similar rates of survival, which are significantly better than patients with TGR 3, 4, and 5, in many studies, these 2 grades have been combined for survival analysis (8-11). In 1999, Ninomiya et al. conducted precise histological examinations of 18 gastric carcinoma patients who underwent gastrectomy after NAC and proposed new TRG criteria for GC, which are now usually referred to as the Japanese Gastric Cancer Association (JGCA) criteria (12). Becker et al. proposed a four-tiered grading system based on large number of GC patients and indicated tumor regression to be an independent prognostic factor of survival $(13,14)$. At present, the most widely used TRG system for evaluating the efficacy of NAC in GC is the one proposed by Ryan et al. in 2005; this system was first applied to surgical specimens of rectal cancer and was later recommended by College of American Pathologists (CAP) as a pathological TRG system for GC (15). In most studies, GC patients who demonstrate histologic complete response or near complete response to NAC have been shown as having better survival (16-19). Whether these patients would benefit from a different regimen to the one administered preoperatively is still controversial.

In this study, according to CAP-TRG, we examined the relationship between clinical data and prognosis among TRG 0 and 1 patients with locally advanced gastric cancer who received NAC after R0 resection and explored the appropriate management of TRG 0 and 1 patients with locally advanced GC after $\mathrm{R} 0$ resection.

We present the following article in accordance with the STROBE reporting checklist (available at http://dx.doi. org/10.21037/atm-20-3986).

\section{Methods}

\section{Patient population}

The retrospective clinical data of TRG 0 and 1 patients with locally advanced GC after R0 Resection who received treatment at The Affiliated Tumor Hospital of Zhengzhou University between January 2012 and December 2018 were included in the study. One hundred and fifty-four patients were enrolled in this study. Patients who met following criteria were included in the study: (I) aged 20-70 years old; (II) ECOG score of 0 or 1; (III) adenocarcinoma confirmed by biopsy specimen; (IV) computed tomography (CT) showed T3-4, N any, M0 or T any, N1-3, M0 before NAC; (V) D2 gastrectomy; (VI) R0 resection; (VII) no history of malignancy; (VIII) non-emergency surgery.

The protocol for the study project was approved by the Ethics Committee of The Affiliated Tumor Hospital of Zhengzhou University (No. 2020002), and it was conducted in accordance with the Declaration of Helsinki, as revised in 2013. Because of the retrospective nature of the research, the requirement for informed consent was waived.

Data on age, gender, serum CEA level, histology, macroscopic type, clinical stage, operation method (laparoscopy or open), operation type (total or distal gastrectomy), combined resection, lymph node metastasis (ypN), TRG, complications, NAC regimen and course, 
Table 1 Criteria of four tumor regression grading systems

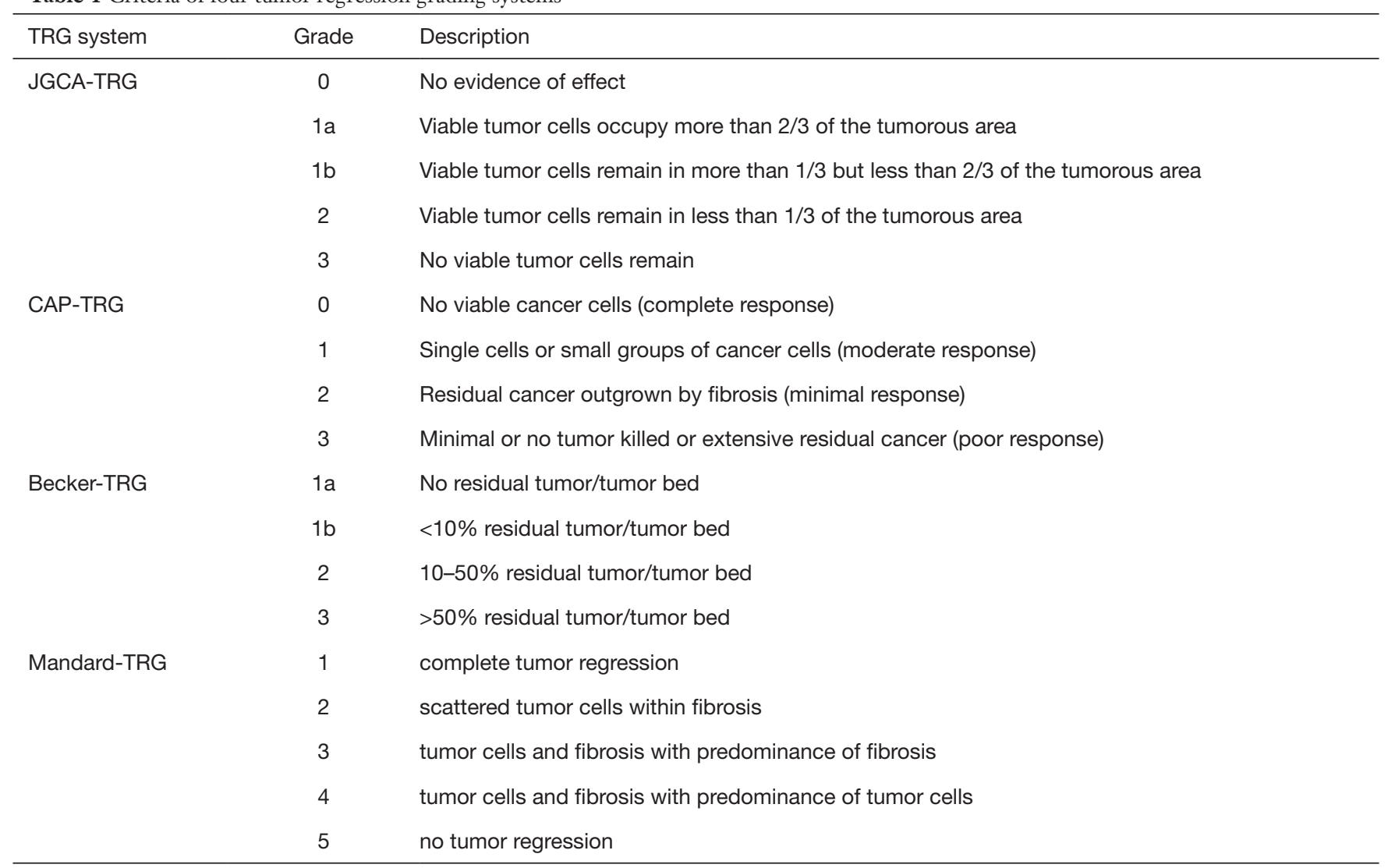

and adjuvant chemotherapy regimen and course were collected. The cut-off value for CEA was $5.0 \mathrm{ng} / \mathrm{mL}$. The patients were categorized according to the Clavien-Dindo classification system (20). If a patient experienced two or more complications, the level of analysis was determined by the highest ranked complication. Complications of grades III-V were considered to be major complications.

\section{Staging}

The chests and abdomens of all of the patients were examined by CT scan. Clinical tumor stage was evaluated by two experienced roentgenologists, according to the eighth edition of the UICC staging system.

\section{Chemotherapy regimen}

The chemotherapy regimens were: (I) monotherapy: S-1and Capecitabine; (II) doublet chemotherapy: SOX, XELOX, and mFOLFOX6; and (III) triplet regimen: DOX, DCF, ECF, and FLOT. The chemotherapeutic dosage was adjusted according to the level of toxic reaction.

\section{Surgery procedure}

Between 3 and 4 weeks after NAC, all patients with no clear surgical contraindications were underwent radical gastrectomy with D2 lymphadenectomy according to the Japanese classification of gastric carcinoma (ver. 4) (21). After the gastrectomy, the specimen was removed through a small median abdominal incision under the xiphoid (about 6-8 cm), and reconstruction was performed in laparoscopy surgery. In open surgery, an incision approximately $20-25 \mathrm{~cm}$ in length was made from the xiphoid to the periumbilical area.

\section{Pathological response}

Pathological response was reevaluated by two pathologists in line with the tumor regression grading (TRG) system (15) as follows: TRG 0: no cancer cells, including lymph nodes (Figure 1A); TRG 1: single cells or small groups of cancer 



Figure 1 Images showing the different stages of the tumor regression grading system. (A) No cancer cells, lymphocytes, and histiocyte infiltration; (B) small groups of cancer cells, lymphocytes and histiocyte infiltration; (C) residual cancer outgrown by fibrosis; (D) minimal or no treatment effect. ( $\mathrm{H} \& \mathrm{E}$, original magnification $\times 100$ ).

cells (Figure 1B); TRG 2: Residual cancer outgrown by fibrosis (Figure 1C), and TRG 3: minimum or no treatment effect and extensive residual cancer cells (Figure 1D).

\section{Follow-up}

Overall survival (OS) was defined as the period from the initiation of NAC to death from any cause, and recurrencefree survival (RFS) was defined as the period from the initiation of NAC to recurrence or death from any cause. All of the patients were followed up every 3 to 6 months. Clinical examination and hematologic analysis were performed at each follow-up visit, including tumor marker assays for carcinoembryonic antigen (CEA), carbohydrate antigen 72-4 (CA72-4), and carbohydrate antigen 19-9 (CA19-9). Abdominal CT was performed every 6 months or when recurrence was clinically suspected. The cut-off date for overall survival was in June 2019, and the median follow-up was 34.1 (range, 6.6-90.9) months. Six patients $(3.9 \%)$ were lost at follow-up.

\section{Statistical analysis}

For continuous variables, data presented as the mean \pm SD and, for categorical variables, as proportions. For univariate analysis, differences in RFS between groups were evaluated by the log-rank test. A Cox proportional hazards model was used to perform multivariate analysis. Covariates with a $\mathrm{P}$ value $<0.1$ in the univariate analysis were included in the multivariate model. A $\mathrm{P}$ value $<0.05$ was considered to represent statistical significance. IBM SPSS Statistics, version 22 (IBM corporation, North Castle Drive, Armonk, NY, USA) was used to conduct all of the analyses.

\section{Results}

\section{Patient characteristics}

A total of 154 patients who underwent NAC combined with radical surgery for GC were ultimately included in this study. Their clinicopathological factors and treatment details are shown in Table 2. Most patients were male (78.6\%), with ages ranging between 26 and 70 (median: 56 years old). The CEA level of 16 patients (10.4\%) after NAC was positive. A majority of patients displayed differentiated histology $(81.8 \%)$ and presented with Borrmann III/IV (58.4\%). Before NAC, 40 (26.0\%), 98 (63.6\%), and $16(10.4 \%)$ patients were classified as stage II, III and IV (clinical stage), respectively. A majority of patients received doublet chemotherapy $(76.0 \%)$ as the regimen of NAC and their courses ranged from 2 to 6 cycles (median: 4). Open surgery, total gastrectomy, and combined resection were received by 110 (71.4\%), 129 
Table 2 The association between clinicopathological factors and treatments and recurrence-free survival

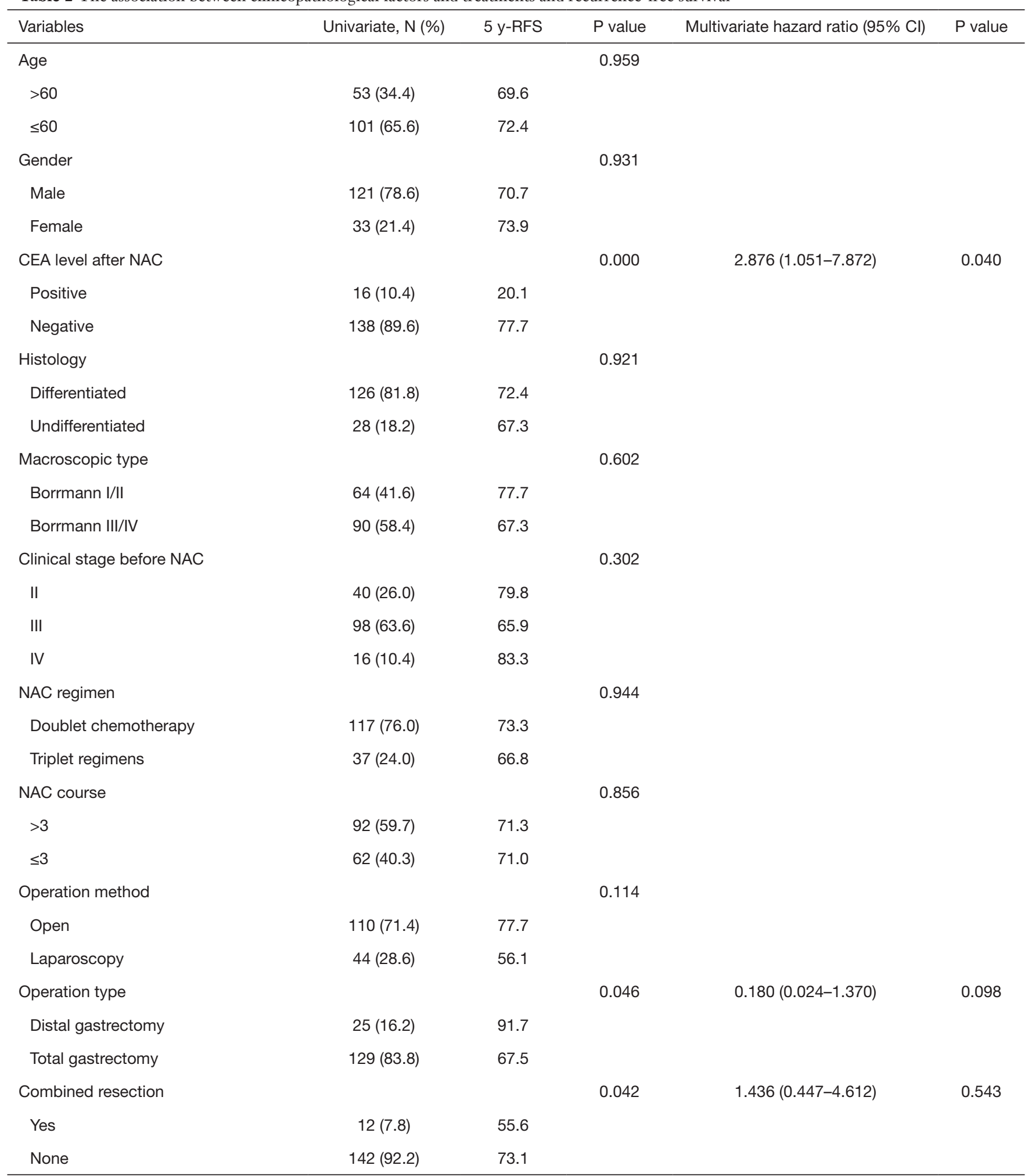

Table 2 (continued) 
Page 6 of 12

Ma et al. Optimal management of locally advanced gastric cancer patients with TRG 0 and 1 after R0 resection

Table 2 (continued)

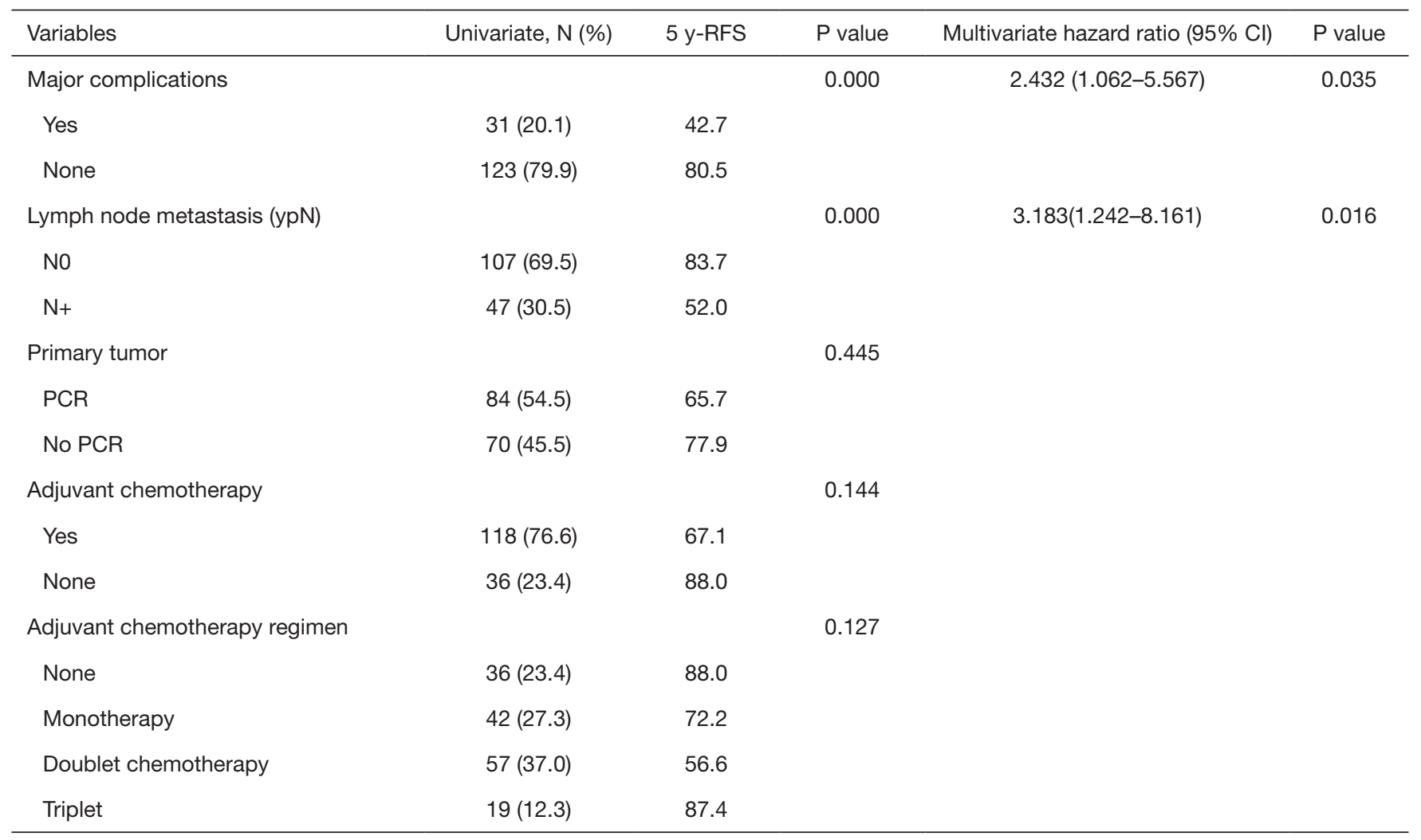

RFS, recurrence-free survival; CEA, carcinoembryonic antigen; NAC, neoadjuvant chemotherapy.

$(83.8 \%)$ and $12(7.8 \%)$ of the patients, respectively. Major complications were experienced by 31 patients $(20.1 \%)$. After surgery, 107 patients (69.5\%) had no lymph node metastasis (ypN0), and in 84 patients (54.5\%) no cancer cells were discovered in the primary tumor. Adjuvant chemotherapy was administered to 118 patients (76.6\%), 42 patients $(27.3 \%)$ received oral drugs alone, 57 patients (37.0) received doublet chemotherapy, and 19 patients (12.3) received three-drug chemotherapy.

\section{Factors associated with survival}

After 34.1 (range, 6.6-90.9) months of follow-up, 27 patients had experienced relapse, 12 of whom died (including 2 patients who died of non-neoplastic causes). The 5-year RFS (Figure 2A) and 5-year OS (Figure 2B) were 71.6\% (95\% CI: 68.5-79.6) and 82.9\% (95\% CI: 76.9-86.1) for the entire cohort, respectively. The univariate analysis revealed that patients with CEA $<5.0 \mathrm{ng} / \mathrm{ml}$ after NAC (77.7\% vs. $20.1 \%, \mathrm{P}<0.001)$, distal gastrectomy $(91.7 \%$ vs. $67.5 \%, \mathrm{P}=0.046$ ) had higher 5-year RFS. Meanwhile, combined resection ( $55.6 \%$ vs. $73.1 \%, \mathrm{P}=0.042)$, major complications $(42.7 \%$ vs. $80.5 \%, \mathrm{P}<0.001)$, and lymph node metastasis $(y p N+)(52.0 \%$ vs. $83.7 \%, \mathrm{P}<0.001)$ had lower 5-year RFS.

The covariates included in Cox proportional hazards model were CEA level after NAC, operation type, combined resection, major complications, and lymph node metastasis $(\mathrm{ypN}+)$. Based on the multivariate analysis, CEA level after NAC (HR =2.876, 95\% CI: 1.051-7.872, $\mathrm{P}=0.040)$, major complications ( $\mathrm{HR}=2.432,95 \% \mathrm{CI}: 1.062-5.567, \mathrm{P}=0.035)$, and lymph node metastasis $(y p N+)(\mathrm{HR}=3.183,95 \% \mathrm{CI}$ : $1.242-8.161, \mathrm{P}=0.016)$ are independent prognostic factors (Table 2).

The 5-year RFS rates for patients with CEA $<5.0 \mathrm{ng} / \mathrm{mL}$ and CEA $\geq 5.0 \mathrm{ng} / \mathrm{mL}$ after NAC were $77.7 \%$ (95\% CI: 74.0-81.4) and 20.1\% (95\% CI: 0-51.6), respectively $(\mathrm{P}=0.000)$ (Figure 2C). The 5-year OS rates for patients with CEA $<5.0 \mathrm{ng} / \mathrm{mL}$ and CEA $\geq 5.0 \mathrm{ng} / \mathrm{mL}$ after NAC were 83.8\% (95\% CI: 74.4-93.2) and 79.8\% (95\% CI: 59.2-100), respectively $(\mathrm{P}=0.102)$ (Figure $2 \mathrm{D})$. The 5 -year RFS rates of patients with and without major complications were 

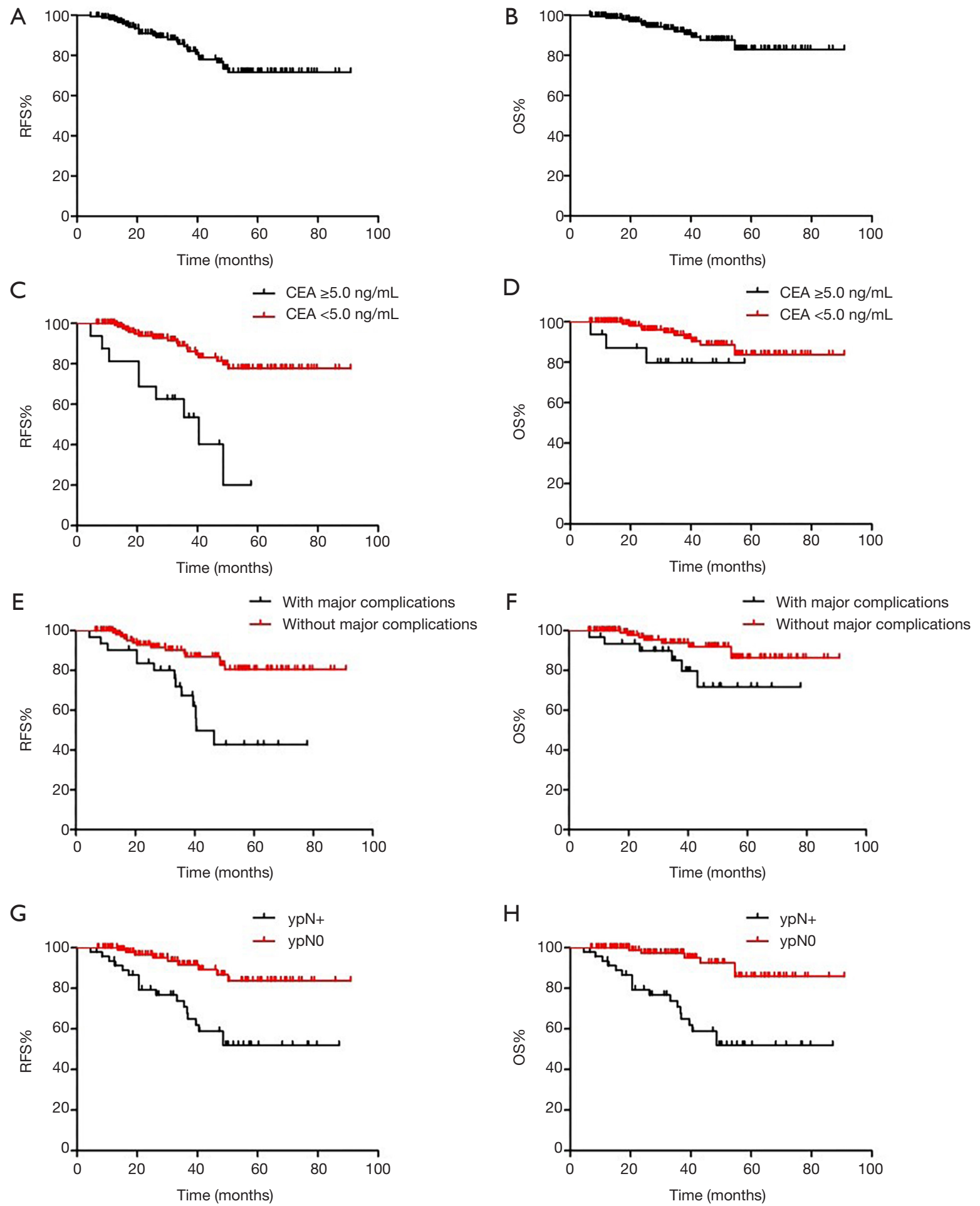

Figure 2 The prognostic value of clinicopathological factors. Kaplan-Meier survival curves for RFS (A) and OS (B) in the overall population. Kaplan-Meier curves for RFS (C) and OS (D) in patients based on CEA level after NAC. Kaplan-Meier curves for RFS (E) and OS (F) in patients based on major complications. Kaplan-Meier curves for RFS (G) and OS (H) in patients based on lymph node metastasis (ypN). RFS, recurrence-free survival; OS, overall survival; NAC, neoadjuvant chemotherapy. 
42.7\% (95\% CI: 20.0-65.4) and 80.5\% (95\% CI: 70.7-90.3), respectively $(\mathrm{P}=0.000)$ (Figure $2 E)$. The 5 -year OS rates of patients with and without major complications were $71.8 \%$ (95\% CI: 50.8-92.8) and 86.4\% (95\% CI: 76.8-96.0), respectively $(\mathrm{P}=0.047)$ (Figure $2 F)$. The 5 -year RFS rates of ypN0 and ypN+ were $83.7 \%$ (95\% CI: 73.1-94.3) and 52.0\% (95\% CI: 35.3-68.7), respectively $(\mathrm{P}=0.000)$ (Figure 2G), The 5-year OS rates of ypN0 and ypN+were $85.9 \%(95 \%$ CI: 74.7-97.1) and 77.6\% (95\% CI: 63.7-91.5), respectively $(\mathrm{P}=0.044)$ (Figure 2H).

\section{Discussion}

At present, NAC is widely used in the standardized treatment of GC, using effective methods to accurately evaluate its efficacy is particularly important. TRG system is mainly to observe the degree of fibrosis of tumor tissues and the proportion of residual tumor cells. TRG system may reflect different efficacy of NAC, help formulate followup treatment regimen and predict prognosis. Many studies showed GC patients who demonstrate histologic complete response or near complete response to NAC have better survival (16-19). In this study, according to CAP-TRG, TRG 0 and 1 patients with local GC after R0 resection following NAC were found to have a good prognosis, which is similar to findings of previous studies $(11,22)$. We also found CEA level after NAC, major complications, and lymph node metastasis $(\mathrm{ypN}+)$ to be independent prognostic factors. However, baseline characteristics and pre-surgical/surgical/post-surgical treatment do not guarantee recurrence-free long-term survival.

CEA is a serum tumor marker that has been used to diagnose and monitor of gastrointestinal malignancies for a long time, and its prognostic ability has been reported many times (23-25). In most previous reports, only preoperative or postoperative levels of CEA were evaluated. In our study, we found that CEA level after NAC has prognostic significance for the long-term survival of TRG 0 and 1 patients with locally advanced GC after R0 resection. A clinical evaluation by Sun and Zhang suggested that high preoperative serum levels of CEA are associated with a higher risk of death, and may predict clinical disease progression after NAC (26); however, the mechanisms of this have not been clarified. This is perhaps due to the association serum CEA concentration has with factors such as tumor differentiation, degree of vascular invasion, and location of CEA expression within cancer cells $(27,28)$.

Previous data has suggested that long-term survival following curative gastrectomy is conversely affected by postoperative intra-abdominal infectious complications (29). Hayashi et al. demonstrated that postoperative infectious complications increase disease recurrence in GC patients (30). In this study, we found that major complications are poor prognostic factors. This outcome can be attributed to several factors. Firstly, the adaptive immune system can protect the host against cancer cells while inflammatory cytokines such as interleukin 1, 6, and 8 (IL-1/6/8) and tumor necrosis factor alpha (TNF- $\alpha$ ) may hinder this process (31). Secondly, inflammation promotes the proliferation and survival of malignant cells (32). Thirdly, anastomotic leakage causes the deposition and implantation of viable exfoliated tumor cells in the enterocoelia, which may increase the local recurrence rate (33). Furthermore, complications always postpone the adjuvant chemotherapy.

$\mathrm{Xu}$ et al. discovered that $\mathrm{ypN}$ was significantly associated with survival, while postsurgical $\mathrm{T}$ category failed to be an independent factor of OS and DFS in patients after perioperative chemotherapy (8). In previous studies, gastric patients with residual cancer cells in the lymph nodes and no cancer cells in the primary tumor after NAC have shown worse survival than patients with no cancer cells in the primary tumor and lymph nodes (2). In this study, lymph node metastasis $(\mathrm{ypN}+)$ was another independent factor of poor prognosis, and can be considered as one of the most important prognostic variables for GC. The response of the primary tumor and lymph nodes to NAC was not identical. Pathological tumor response assesses the effects of chemotherapy by focusing attention on the fibrosis primary lesion but affording insufficient attention on the changes of lymph node.

The TNM system is well known as an effective predictor of the prognosis of GC patients. Although pre-operative TNM staging by CT scans and post-operative staging by pathology are not in perfect agreement, clinical TNM staging is crucial for planning appropriate treatment and predicting the survival of GC patients without preoperative treatment $(34,35)$. In our study, no correlation was found between the baseline characteristics and RFS. Kurokawa et al. reported that histological response validated the response assessment criteria for overall survival in NAC for GC, better than RECIST or TNM (36). Cho et al. reported 22 patients who achieved pCR after NAC had a good prognosis (22), with an overall survival rate at 5 years of $85 \%$; however 19 of the subjects had either stage III or IV disease based on the $7^{\text {th }}$ TNM edition, 2 were positive 
for peritoneal cytology, and 12 did not receive adjuvant chemotherapy. In our study, there was no correlation discovered between RFS and pre-surgical, surgical, or postsurgical treatments. As such, we can gather that tumor load or treatment are not predictive of long-term survival in TRG 0 and 1 patients after NAC. The biology of GC with TRG 0 and 1 with NAC is likely to be distinct from nonTRG 0 and 1 tumors, and this is reflected in the prognosis.

The non-operative management (NOM) strategy was first put forward by Habr-Gama et al. (37), who found that rectal cancer patients achieved clinical complete response to neoadjuvant chemoradiotherapy and total mesorectal excision is no longer required. NOM may preserve sphincter function and avoid surgery-related complications, and is a new norm in the treatment of rectal cancer. As far as we know, no similar reports on GC exist. Several problems impelled us to carry out similar clinical research on GC. Firstly, GC patients achieving pCR after $\mathrm{NAC}$ is uncommon in clinical practice, the pCR rate were respectively $13.0 \%, 10.0 \%$ and $16.0 \%$ by using the ECF(like) (38), DCF (39), and FLOT (6) regimen (6). Next, clinical response seems to correlate poorly with pathology in preoperative staging of gastric adenocarcinoma after NAC, Thirdly, pathological response has a higher validity than radiography (36).

Previous studies have revealed brain metastasis from GC occurs in less than $1 \%$ of clinical cases $(40,41)$. Fields et al. reported the incidence of brain metastasis in pCR patients to be $8 \%$, and one-third of the metastases experienced by pCR patients were brain metastases (42). Due to bloodbrain barrier, penetration of chemotherapeutic agents into central nervous system (CNS) is diminished (43). As a result, TRG 0 and 1 patients may have increased risk of brain recurrence, and CNS symptoms in these patients should be acknowledged.

Since heterogeneity exists in GC cells, the identification of predictive biomarkers may make a better prognosis in patients more likely. A new molecular classification was proposed, which divided GC into four subtypes: MSI tumors, EBV-infected tumors, genomically stable tumors, and chromosomally unstable tumors (44). EBV-infected tumors are associated with better prognosis (45). Several studies have demonstrated the expression of LGR5, FZD7 to represent a worse prognosis in GC $(46,47)$.

Nevertheless, there is a possibility that the results were influenced by several factors. Firstly, the inherent limitations of this study's retrospective nature may have introduced selection bias, although we applied up inclusion criteria to take this into account. Secondly, the relatively small number of patients and the low incidence of TRG 0 and 1 may have limited the power of the study. Finally, the variety in chemotherapy regimens is another potential limitation.

\section{Conclusions}

In conclusion, TRG 0 and 1 patients with locally advanced GC after R0 resection following NAC had a good prognosis. The surgical procedure and perioperative care should be approached with caution to avoid causing complications. Monotherapy or no chemotherapy may serve as treatment options for TRG 0 and 1 patients while avoiding adverse prognostic factors. Further research on the predictive and prognostic factors for TRG 0 and 1 patients, such as the role of definition of EBV, early 18-FDG-PET assessment, MMR, and microsatellite status could help with management-related decision-making.

\section{Acknowledgments}

Thank you to the numerous individuals participated in this study.

Funding: This work was funded by 1000 Talents Program of Central plains (No. 204200510023), Science and Technique Foundation of Henan Province (No. 202102310414) and Science and Technique Foundation of Henan Province (No. 192102310311). We declare that the funding agencies had no role in study design, data collection, data analysis, data interpretation, or writing of the manuscript.

\section{Footnote}

Reporting Checklist: The authors have completed the STROBE reporting checklist. Available at http://dx.doi. org/10.21037/atm-20-3986

Data Sharing Statement: Available at http://dx.doi. org/10.21037/atm-20-3986

Conflicts of Interest: All authors have completed the ICMJE uniform disclosure form (available at http://dx.doi. org/10.21037/atm-20-3986). The authors have no conflicts of interest to declare.

Etbical Statement: The authors are accountable for all aspects of the work in ensuring that questions related 
to the accuracy or integrity of any part of the work are appropriately investigated and resolved. The protocol for the research project was approved by the Ethics Committee of The Affiliated Tumor Hospital of Zhengzhou University (No. 2020002) and conformed to the provisions of the Declaration of Helsinki, as revised in 2013. Because of the retrospective nature of the research, the requirement for informed consent was waived.

Open Access Statement: This is an Open Access article distributed in accordance with the Creative Commons Attribution-NonCommercial-NoDerivs 4.0 International License (CC BY-NC-ND 4.0), which permits the noncommercial replication and distribution of the article with the strict proviso that no changes or edits are made and the original work is properly cited (including links to both the formal publication through the relevant DOI and the license). See: https://creativecommons.org/licenses/by-nc-nd/4.0/.

\section{References}

1. Chen $\mathrm{W}$, Zheng R, Baade PD, et al. Cancer statistics in China, 2015. CA Cancer J Clin 2016;66:115-32.

2. Zhang CD, Yamashita H, Seto Y. Gastric cancer surgery: historical background and perspective in Western countries versus Japan. Ann Transl Med 2019;7:493.

3. Wilke H, Preusser P, Fink U, et al. Preoperative chemotherapy in locally advanced and nonresectable gastric cancer: a phase II study with etoposide, doxorubicin, and cisplatin. J Clin Oncol 1989;7:1318-26.

4. Cunningham D, Allum WH, Stenning SP, et al. Perioperative chemotherapy versus surgery alone for resectable gastroesophageal cancer. $\mathrm{N}$ Engl J Med 2006;355:11-20.

5. Ychou M, Boige V, Pignon JP, et al. Perioperative chemotherapy compared with surgery alone for resectable gastroesophageal adenocarcinoma: an FNCLCC and FFCD multicenter phase III trial. J Clin Oncol 2011;29:1715-21.

6. Al-Batran SE, Homann N, Pauligk C, et al. Effect of Neoadjuvant Chemotherapy Followed by Surgical Resection on Survival in Patients With Limited Metastatic Gastric or Gastroesophageal Junction Cancer: The AIOFLOT3 Trial. JAMA Oncol 2017;3:1237-44.

7. Mandard AM, Dalibard F, Mandard JC, et al. Pathologic assessment of tumor regression after preoperative chemoradiotherapy of esophageal carcinoma. Clinicopathologic correlations. Cancer 1994;73:2680-6.
8. Xu X, Zheng G, Zhang T, et al. Is pathologic tumor regression grade after neo-adjuvant chemotherapy a promising prognostic indicator for patients with locally advanced gastric cancer? A cohort study evaluating tumor regression response. Cancer Chemother Pharmacol 2019;84:635-46.

9. Noble F, Lloyd MA, Turkington R, et al. Multicentre cohort study to define and validate pathological assessment of response to neoadjuvant therapy in oesophagogastric adenocarcinoma. Br J Surg 2017;104:1816-28.

10. Kosuga T, Ichikawa D, Otsuji E. Pathologic tumor response to neoadjuvant chemotherapy in gastroesophageal cancer: what does it mean? Transl Gastroenterol Hepatol 2016;1:75.

11. Wong J. Impact of pathologic tumor response in the treatment of gastric cancer. Transl Gastroenterol Hepatol 2016;1:71.

12. Ninomiya Y, Yanagisawa A, Kato Y, et al. Histological indications of a favorable prognosis with far-advanced gastric carcinomas after preoperative chemotherapy. J Cancer Res Clin Oncol 1999;125:699-706.

13. Becker K, Mueller JD, Schulmacher C, et al. Histomorphology and grading of regression in gastric carcinoma treated with neoadjuvant chemotherapy. Cancer 2003;98:1521-30.

14. Becker K, Langer R, Reim D, et al. Significance of histopathological tumor regression after neoadjuvant chemotherapy in gastric adenocarcinomas: a summary of 480 cases. Ann Surg 2011;253:934-9.

15. Ryan R, Gibbons D, Hyland JM, et al. Pathological response following long-course neoadjuvant chemoradiotherapy for locally advanced rectal cancer. Histopathology 2005;47:141-6.

16. Zhu Y, Sun Y, Hu S, et al. Comparison of five tumor regression grading systems for gastric adenocarcinoma after neoadjuvant chemotherapy: a retrospective study of 192 cases from National Cancer Center in China. BMC Gastroenterol 2017;17:41.

17. Derieux S, Svrcek M, Manela S, et al. Evaluation of the prognostic impact of pathologic response to preoperative chemotherapy using Mandard's Tumor Regression Grade (TRG) in gastric adenocarcinoma. Digestive and Liver Disease 2020;52:107-14.

18. Stark AP, Ikoma N, Chiang YJ, et al. Characteristics and Survival of Gastric Cancer Patients with Pathologic Complete Response to Preoperative Therapy. Ann Surg Oncol 2019;26:3602-10.

19. Fokas E, Ströbel P, Fietkau R, et al. Tumor Regression 
Grading After Preoperative Chemoradiotherapy as a Prognostic Factor and Individual-Level Surrogate for Disease-Free Survival in Rectal Cancer. J Natl Cancer Inst 2017. doi: 10.1093/jnci/djx095.

20. Clavien PA, Barkun J, de Oliveira ML, et al. The ClavienDindo classification of surgical complications: five-year experience. Ann Surg 2009;250:187-96.

21. Japanese Gastric Cancer Association. Japanese gastric cancer treatment guidelines 2014 (ver. 4). Gastric Cancer 2017;20:1-19.

22. Cho H, Nakamura J, Asaumi Y, et al. Long-term survival outcomes of advanced gastric cancer patients who achieved a pathological complete response with neoadjuvant chemotherapy: a systematic review of the literature. Ann Surg Oncol 2015;22:787-92.

23. Dawood S. The evolving role of immune oncology in colorectal cancer. Chin Clin Oncol 2018;7:17.

24. Wang W, Seeruttun SR, Fang C, et al. Prognostic Significance of Carcinoembryonic Antigen Staining in Cancer Tissues of Gastric Cancer Patients. Ann Surg Oncol 2016;23:1244-51.

25. Jaklitsch M, Petrowsky H. The power to predict with biomarkers: carbohydrate antigen 19-9 (CA 19-9) and carcinoembryonic antigen (CEA) serum markers in intrahepatic cholangiocarcinoma. Transl Gastroenterol Hepatol 2019;4:23.

26. Sun Z, Zhang N. Clinical evaluation of CEA, CA199, CA72-4 and CA125 in gastric cancer patients with neoadjuvant chemotherapy. World J Surg Oncol 2014;12:397.

27. Koprowski H, Steplewski Z, Mitchell K, et al. Colorectal carcinoma antigens detected by hybridoma antibodies. Somatic Cell Genet 1979;5:957-71.

28. Kim JH, Jun KH, Jung H, et al. Prognostic Value of Preoperative Serum Levels of Five Tumor Markers (Carcinoembryonic Antigen, CA19-9, Alphafetoprotein, CA72-4, and CA125) in Gastric Cancer. Hepatogastroenterology 2014;61:863-9.

29. Wang S, Xu L, Wang Q, et al. Postoperative complications and prognosis after radical gastrectomy for gastric cancer: a systematic review and meta-analysis of observational studies. World J Surg Oncol 2019;17:52.

30. Hayashi T, Yoshikawa T, Aoyama T, et al. Impact of infectious complications on gastric cancer recurrence. Gastric Cancer 2015;18:368-74.

31. Dunn GP, Old LJ, Schreiber RD. The immunobiology of cancer immunosurveillance and immunoediting. Immunity 2004;21:137-48.
32. Mantovani A, Allavena P, Sica A, et al. Cancer-related inflammation. Nature 2008;454:436-44.

33. Bell SW, Walker KG, Rickard MJ, et al. Anastomotic leakage after curative anterior resection results in a higher prevalence of local recurrence. Br J Surg 2003;90:1261-6.

34. Barros RH, Penachim TJ, Martins DL, et al. Multidetector computed tomography in the preoperative staging of gastric adenocarcinoma. Radiol Bras 2015;48:74-80.

35. Lee MH, Choi D, Park MJ, et al. Gastric cancer: imaging and staging with MDCT based on the 7th AJCC guidelines. Abdom Imaging 2012;37:531-40.

36. Kurokawa Y, Shibata T, Sasako M, et al. Validity of response assessment criteria in neoadjuvant chemotherapy for gastric cancer (JCOG0507-A). Gastric Cancer 2014;17:514-21.

37. Habr-Gama A, Perez RO, Nadalin W, et al. Operative versus nonoperative treatment for stage 0 distal rectal cancer following chemoradiation therapy: long-term results. Ann Surg 2004;240:711-7; discussion 777-8.

38. Bichev D, Treese C, von Winterfeld M, et al. High Impact of Histopathological Remission for Prognosis after Perioperative Chemotherapy with ECF and ECFLike Regimens for Gastric and Gastroesophageal Adenocarcinoma. Oncology 2015;89:95-102.

39. Ferri LE, Ades S, Alcindor T, et al. Perioperative docetaxel, cisplatin, and 5-fluorouracil (DCF) for locally advanced esophageal and gastric adenocarcinoma: a multicenter phase II trial. Ann Oncol 2012;23:1512-7.

40. Go PH, Klaassen Z, Meadows MC, et al. Gastrointestinal cancer and brain metastasis: a rare and ominous sign. Cancer 2011;117:3630-40.

41. Kasakura Y, Fujii M, Mochizuki F, et al. Clinicopathological study of brain metastasis in gastric cancer patients. Surg Today 2000;30:485-90.

42. Fields RC, Strong VE, Gonen M, et al. Recurrence and survival after pathologic complete response to preoperative therapy followed by surgery for gastric or gastrooesophageal adenocarcinoma. Br J Cancer 2011;104:1840-7.

43. Chabner BA, Longo DL. Cancer chemotherapy and biotherapy: principles and practice. Lippincott Williams \& Wilkins, 2011.

44. Cancer Genome Atlas Research Network. Comprehensive molecular characterization of gastric adenocarcinoma. Nature 2014;513:202-9.

45. Hewitt LC, Inam IZ, Saito Y, et al. Epstein-Barr virus and mismatch repair deficiency status differ between oesophageal and gastric cancer: A large multi-centre study. 
Eur J Cancer 2018;94:104-14.

46. Xi HQ, Cai AZ, Wu XS, et al. Leucine-rich repeatcontaining G-protein-coupled receptor 5 is associated with invasion, metastasis, and could be a potential therapeutic target in human gastric cancer. Br J Cancer
2014;110:2011-20.

47. Li G, Su Q, Liu H, et al. Frizzled7 Promotes Epithelialto-mesenchymal Transition and Stemness Via Activating Canonical Wnt/beta-catenin Pathway in Gastric Cancer. Int J Biol Sci 2018;14:280-93.

Cite this article as: Ma F, Zhang Y, Peng L, Zhang Z, Yang W, Chai J, Zhang B, Ji S, Hua Y, Chen X, Luo S. Which management strategy is appropriate for locally advanced gastric cancer patients with TRG 0 and 1 after R0 resection? Ann Transl Med 2020;8(15):948. doi: 10.21037/atm-20-3986 